\title{
Multiparametric analytical quantification of materials at nanoscale in tapping force microscopy
}

Farokh Payam, A., Morelli, A., \& Lemoine, P. (2021). Multiparametric analytical quantification of materials at nanoscale in tapping force microscopy. Applied Surface Science, 536, [147698]. https://doi.org/10.1016/j.apsusc.2020.147698

Link to publication record in Ulster University Research Portal

\section{Published in:}

Applied Surface Science

Publication Status:

Published (in print/issue): 15/01/2021

DOI:

10.1016/j.apsusc.2020.147698

\section{Document Version}

Author Accepted version

\section{General rights}

Copyright for the publications made accessible via Ulster University's Research Portal is retained by the author(s) and / or other copyright owners and it is a condition of accessing these publications that users recognise and abide by the legal requirements associated with these rights.

\section{Take down policy}

The Research Portal is Ulster University's institutional repository that provides access to Ulster's research outputs. Every effort has been made to ensure that content in the Research Portal does not infringe any person's rights, or applicable UK laws. If you discover content in the Research Portal that you believe breaches copyright or violates any law, please contact pure-support@ulster.ac.uk. 


\title{
Multiparametric Analytical Quantification of Materials at Nanoscale in Tapping Force Microscopy
}

\author{
Amir Farokh Payam, Alessio Morelli, Patrick Lemoine
}

Nanotechnology and Integrated Bioengineering Centre (NIBEC), School of Engineering, Ulster University, Newtownabbey BT37 0QB, UK

Email: a.farokh-payam@ulster.ac.uk

\begin{abstract}
Atomic force microscopy (AFM) is a powerful technique for accurate, reliable and non-destructive imaging and characterization of materials at the nanoscale. Among the numerous AFM methods, amplitude modulation or tapping mode AFM (AM-AFM) is an established method for imaging and characterization for most commercial AFM systems. Despite its high spatial resolution and sensitivity, quantitative characterization by AM-AFM lag behind other advanced AFM methods as far as quantification of materials properties is concerned. In this paper a fully analytical multiparametric approach for AM-AFM is proposed which simultaneously quantifies the Hamaker constant and viscoelastic properties of materials. The main advantage of the proposed method lies in the inclusion of adhesion to calculate viscoelasticity, which makes it superior to the current equations used in the AFM community. The accuracy of the proposed method is validated by several simulations and experiments and comparison with nanoindentation results, which strongly support its candidacy as a method of choice for material properties quantification by dynamic AFM.
\end{abstract}


Quantification and characterization of adhesive and viscoelastic properties of materials at atomic and nanoscale range is an important topic in the fields of chemistry, physics, biology, polymer, composite and materials science and engineering ${ }^{1-7}$. To provide a framework for identification and quantification of materials properties at nanometer scale, development in the integration of nanoscale instrumentation with advances in data analysis and computational methodologies is needed. Atomic force microscopy (AFM), being a versatile technique for characterization and imaging with angstrom resolution, provides the possibility for quantification of materials properties from observable data. AFM is used to calculate the Hamaker constant of materials as a quantitative measure of attractive van der Waals (vdW) interaction between the cantilever-tip ensemble and samples ${ }^{8-12}$. For the calculation of the elastic and viscoelastic properties of materials, such as stiffness, viscosity and loss tangent, different methods based on the force-curves, dissipation and virial concepts and multifrequency approaches have been proposed ${ }^{13-27}$. By applying bimodal AFM in air environment the stiffness and Hamaker constant of materials could be simultaneously quantified ${ }^{15}$. However, this method cannot provide information about the viscoelastic properties of the sample. Bengalia et al. ${ }^{20}$ quantified the viscoelastic properties of polymers including stiffness, viscosity and loss tangent by applying bimodal AFM. Thoren et al. ${ }^{18}$ proposed a dynamic AFM based on intermodulation technique to measure the elastic and viscous forces. The viscoelasticity of polymers and cells are measured using the force curves of tapping mode AFM ${ }^{19,26}$. However, in all of these methods the Hamaker constant of the samples, cannot be calculated. Also, to calculate the stiffness of the materials, all of these methods neglect the effect of adhesion in viscoelastic calculation and mostly are based on Hertz contact mechanics model ${ }^{28-31}$ which can be a source of error especially when the adhesion of the sample surface is considerable. Besides, multifrequency techniques include bimodal and intermodulation methods require extra equipment such as additional controllers and lock-in amplifiers.

AM-AFM AFM, one of the most common techniques of force microscopy, has widespread applications in nanoscale characterization, molecular and atomic scale imaging and spectroscopy ${ }^{32-35}$. The energy dissipation process of the interaction between cantilever tip ensemble and sample surface can be measured and quantified from phase images of tapping mode AFM ${ }^{34}$. However, in tapping mode force microscopy, the interaction force between tip and sample cannot directly be measured and needs to be reconstructed from the deflection signal in time domain ${ }^{36-39}$ or measured amplitude/phase shift versus distance curves as the oscillating cantilever is brought closer to the sample surface called as minimum distance of approach methods ${ }^{31,40-45}$. For time domain methods, presence of noise is the main challenge which makes their implementation complex. On the other hand, all the minimum distance of approach methods is based on numerical integration equations. Also, in all these methods, the chemical and mechanical parameters such as Hamaker constant and Young modulus cannot be calculated directly. In other words, until now, there is not any analytical equation for the direct extraction of the mechanical, chemical and viscoelastic parameters simultaneously from measured data for tapping mode AFM. In this paper, fully analytical expressions for direct calculation of effective Hamaker constant, Young modulus and viscoelasticity are proposed, based on DMT force model (for conservative regime) and Kelvin-Voigt model (for dissipative regime). The main advantages of the proposed method are direct access to the mechanical/chemical and viscoelastic parameters of interaction and analytical quantification of the interaction force from measured data plus its broad applicability for different cantilevers, free amplitudes and samples from rigid to compliant. 


\section{Theory}

The proposed method consists in the application of physical principles to develop the analytical equations to retrieve the Hamaker constant and the viscoelastic properties of the sample. As illustrated in figure 1, the observable amplitude $(A)$ and phase $(\phi)$ are acquired as a function of the average tip-surface distance $z_{c}$ (top-left) which is subsequently converted into the closest distance between tip and sample $d$ (top-middle). By the resulting phase-distance curve, the intermolecular distance between tip and the atoms of the surface $\left(a_{0}\right)$ defined as the distance of change from fully attractive to repulsive regime, coinciding with the local maximum is obtained (topcenter). By applying the proposed analytical equations, and having previous knowledge of the probe's characteristics, the material's properties are extracted from the data (top-right). Finally, the obtained parameters are utilized to reconstruct the force-distance curve according to DMT theory (bottom right).


Figure 1. Schematic of proposed method. The probe interaction with the sample's surface (bottom left) is acquired in the form of amplitude and phase vs average distance between tip and sample (top left). Calculating the closest distance from the amplitude of cantilever oscillation and the average separation distance (middle center), the curves are converted (top center). By applying the proposed equations on the observable data using the known cantilever parameters, the effective Hamaker constant and Young modulus are calculated (top right). Using the definition of energy dissipation and virial, the resonance frequency of cantilever and the obtained Young modulus, the coefficient of viscosity is calculated (up right). Finally, the calculated parameters are used to reconstruct the force based on DMT force model (bottom right).

Based on the definition of energy conservation and virial theorem, over one period of oscillation the following analytical equations relating the observables to the tip-surface force parameters can be obtained ${ }^{46}$

$$
\begin{aligned}
& V_{t s}=\frac{1}{T} \int_{0}^{T_{0}} F_{t s}(d) z(t) d t=-\frac{k A A_{0}}{2 Q} \cos (\phi) \\
& E_{\text {diss }}=\int_{0}^{T_{0}} F_{t s}(d) \dot{z}(t) d t=-\frac{\pi k A}{Q}\left(A-A_{0} \sin (\phi)\right)
\end{aligned}
$$


Were $z(t)=A \cos (\omega t+\phi)$ is the instantaneous tip deflection, $A$ and $\phi$ are amplitude and phase of signal, $k$ and $Q$ are spring constant and quality factor of cantilever, respectively. $F_{t s}$ is the interaction force and $A_{0}$ is the free amplitude.

On the other hand, the conservative and non-conservative interaction force can be described by the following, according to the DMT and Kelvin-Voigt models ${ }^{20,47}$

$$
\begin{gathered}
F_{t s c}= \begin{cases}-\frac{H R}{6 d^{2}} & d \geq a_{0} \\
-\frac{H R}{6 a_{0}^{2}}+\frac{4 E_{e f f} \sqrt{R}}{3}\left(a_{0}-d\right)^{3 / 2} & d<a_{0}\end{cases} \\
F_{t s n c}= \begin{cases}0 & d \geq a_{0} \\
-\eta \sqrt{R\left(a_{0}-d\right)} \dot{d} & d<a_{0}\end{cases}
\end{gathered}
$$

Where $R$ is the tip radius, $a_{0}$ is the intermolecular distance, $H$ is Hamaker constant, $\eta$ is the coefficient of viscosity, $E_{\text {eff }}$ is the effective tip/surface Young modulus defined as $E_{\text {eff }}=\left[\left(1-v_{s}^{2}\right) / E_{s}+\left(1-v_{t}^{2}\right) / E_{t}\right]^{-1}$, where $E_{s}$, $E_{t}, v_{s}$ and $v_{t}$ are Young modulus and poison ratio of sample and tip, respectively. Combining equations (1) and (3) for the case $d \geq a_{0}$, the following equation results ${ }^{34}$

$$
V_{t s}=\frac{1}{T} \int_{0}^{T_{0}}-\frac{H R}{6 d^{2}} z(t) d t \approx \frac{H R}{6 A}\left[\left(\frac{d+A}{A}\right)^{2}-1\right]^{-3 / 2}
$$

Based on the equations (1) and (5), the analytical expression to calculate the Hamaker constant is obtained

$H=-\frac{3 k A_{0} A^{2} \cos (\phi)}{Q R}\left[\left(\frac{d+A}{A}\right)^{2}-1\right]^{3 / 2}$

On the other hand, for the case that $d<a_{0}$, combining equations (1) and (3) with the assumption that the oscillation amplitude is considerably larger than the indentation length of the tip into the sample $\left(A \gg a_{0}-d=\right.$ $\delta$ ) gives (details are given in supplementary information)

$$
E_{\text {eff }}=\frac{k}{Q\left(a_{0}-d\right)^{2}} \sqrt{\frac{2 A\left(A_{0}^{2}-A^{2}\right)}{R}}+\frac{\mathrm{H} \sqrt{\mathrm{R}}}{8 \mathrm{a}_{0}^{2}\left(\mathrm{a}_{0}-\mathrm{d}\right)^{3 / 2}}
$$

Where $H$ is calculated from (6). Hence the following equation can be obtained for the parameters of conservative interaction force in AM-AFM

$$
\left\{\begin{array}{c}
H=-\frac{3 k A_{0} A^{2} \cos (\phi)}{Q R}\left[\left(\frac{d+A}{A}\right)^{2}-1\right]^{3 / 2} \quad d \geq a_{0} \\
E_{e f f}=\frac{k}{Q\left(a_{0}-d\right)^{2}} \sqrt{\frac{2 A\left(A_{0}^{2}-A^{2}\right)}{R}}+\frac{\mathrm{H} \sqrt{\mathrm{R}}}{8 \mathrm{a}_{0}^{2}\left(\mathrm{a}_{0}-\mathrm{d}\right)^{3 / 2}} d<a_{0}
\end{array}\right.
$$

Finally, by replacement of calculated effective Young modulus and Hamaker constant in equation (3), the conservative interaction force based on DMT model can be reconstructed.

To calculate the viscoelastic properties of materials, the virial and energy dissipation can be written $\operatorname{as}^{20}$ : 
$V_{t s}=-\frac{1}{\pi} \int_{0}^{\delta_{\max }} k_{t s}(\delta) \sqrt{2 A} \sqrt{\delta_{\max }-\delta} d \delta$

$E_{\text {diss }}=-2 \int_{0}^{\delta_{\max }} \eta \omega_{0} \sqrt{R \delta} \sqrt{2 A} \sqrt{\delta_{\max }-\delta} d \delta$

Where, $\delta=\left(a_{0}-d\right)$ is the indentation, $\delta_{\max }$ is the maximum indentation and $k_{t s}=d F_{c t s} / d \delta$.

Based on the mathematical approach presented in ${ }^{20}$, the solution of equations (9) and (10) are given as:

$V_{t s}=-0.25 E_{e f f} \sqrt{2 A R} \delta_{\max }^{2}$

$E_{\text {diss }}=-\frac{\pi}{4} \sqrt{2 A R} \eta \omega_{0} \delta_{\max }^{2}$

From equations (11) and (12), the viscosity coefficient of equation (4) is obtained:

$\eta=\frac{2 E_{e f f} E_{d i s s}}{\left(2 \pi \omega_{0}\right) V_{t s}}$

From the definition of loss tangent ${ }^{16,17}$, the retardation time of Kelvin-Voigt model is described as:

$\tau=\frac{\tan \rho}{\omega_{0}}=\frac{\eta}{2 E_{e f f}}$

As it can be seen from (13) and (14), viscosity and retardation time in our model depend on the frequency of the cantilever, meaning that working with experimental data, the obtained values will vary with the excitation frequency. The argument will be discussed in more detail in the results and discussion section.

\section{Materials and Methods}

\subsection{Simulation}

The numerical solution of AFM equations was calculated using a fourth order Runge-Kutta algorithm. The simulation has been performed for the cantilevers and samples with the following parameters:

$k=30 \frac{N}{m}, Q=300, f_{0}=300 \mathrm{kHz}, R=5 \mathrm{~nm}, A_{0}=10 \mathrm{~nm}$.

Being the tip-sample interface characterized by Young modulus, Hamaker constant and viscosity, in order to evaluate the accuracy of equations over different materials from rigid to compliant, three different cases are considered:

Case1: $E_{\text {eff }}=10 \mathrm{GPa}, H=10^{-19} \mathrm{~J}, \eta=100$ Pa.s.

Case2: $E_{e f f}=1 \mathrm{GPa}, H=10^{-20} \mathrm{~J}, \eta=50$ Pa.s.

Case3: $E_{\text {eff }}=100 \mathrm{MPa}, H=10^{-20} \mathrm{~J}, \eta=10 \mathrm{~Pa} . \mathrm{s}$. 


\subsection{Experiments}

For the experimental verification of the present model two samples of material with markedly different Young modulus were employed: polystyrene (PS - Agar Scientific) and low-density polyethylene (LDPE - Goodfellow).

The experiments were performed in air using a commercial AFM system (D3100 Nasoscope III Digital Instruments, now Bruker) in AM-AFM, with a silicon probe for tapping mode (RTESPA Bruker), having spring constant $28.8 \mathrm{Nm}^{-1}$ (calculated by Sader method ${ }^{48}$ ), first resonance frequency $297.6 \mathrm{kHz}$ and quality factor of about 470. Prior to each set of experiments, the cantilever was tuned at the desired amplitude at a lift height of $100 \mathrm{~nm}$, recording resonance frequency and quality factor to be used for the calculations. The experiments were performed for each sample tuning the amplitude of resonance at $1 \mathrm{~V}$ and $0.5 \mathrm{~V}$ (driven at the first resonance frequency), and acquiring arrays of $5 \times 5$ curves of amplitude, phase and deflection while ramping the $\mathrm{Z}$ piezo of the AFM. After termination of experiments on each sample, the tip radius of curvature was estimated by acquisition of a $1 \mathrm{x} 1 \mu \mathrm{m}^{2}$ image on a qualification sample (TipCheck sample, BudgetSensors.com) and processing by tip characterization procedure with SPIP software, with estimations of $10.5 \mathrm{~nm}$ (PS) and $17.5 \mathrm{~nm}$ (LDPE). On completion of a full set of experiments on both samples, the amplitude sensitivity has been measured by amplitude curve calibration on qualification sample, resulting in a conversion factor of $36.1 \mathrm{~nm} / \mathrm{V}$.

Nanoindentation measurements were also carried out using a Nanoidenter XP with a diamond Berkovitch tip using the continuous stiffness measurement (CSM) option. The indenter tip was calibrated using the Oliver and Pharr method. The indentations were carried out with a residual drift criterion of $0.1 \mathrm{~nm} / \mathrm{s}$ at a constant strain rate of 0.1 $\mathrm{s}^{-1}$ up to $2000 \mathrm{~nm}$ depth.

\section{Results and Discussion}

Figure 2 shows the simulation results of calculated Hamaker constant, Young modulus and coefficient of viscosity for three different materials ranges from rigid (case 1) to semi-rigid (case 2) and compliant material (case 3). Furthermore, the comparison between obtained viscoelasticity parameters from our method and Hertz model are given in figures 2 (b), (c), (e), (f), (h) and (i). More details of simulations are given in Supplementary Information. 

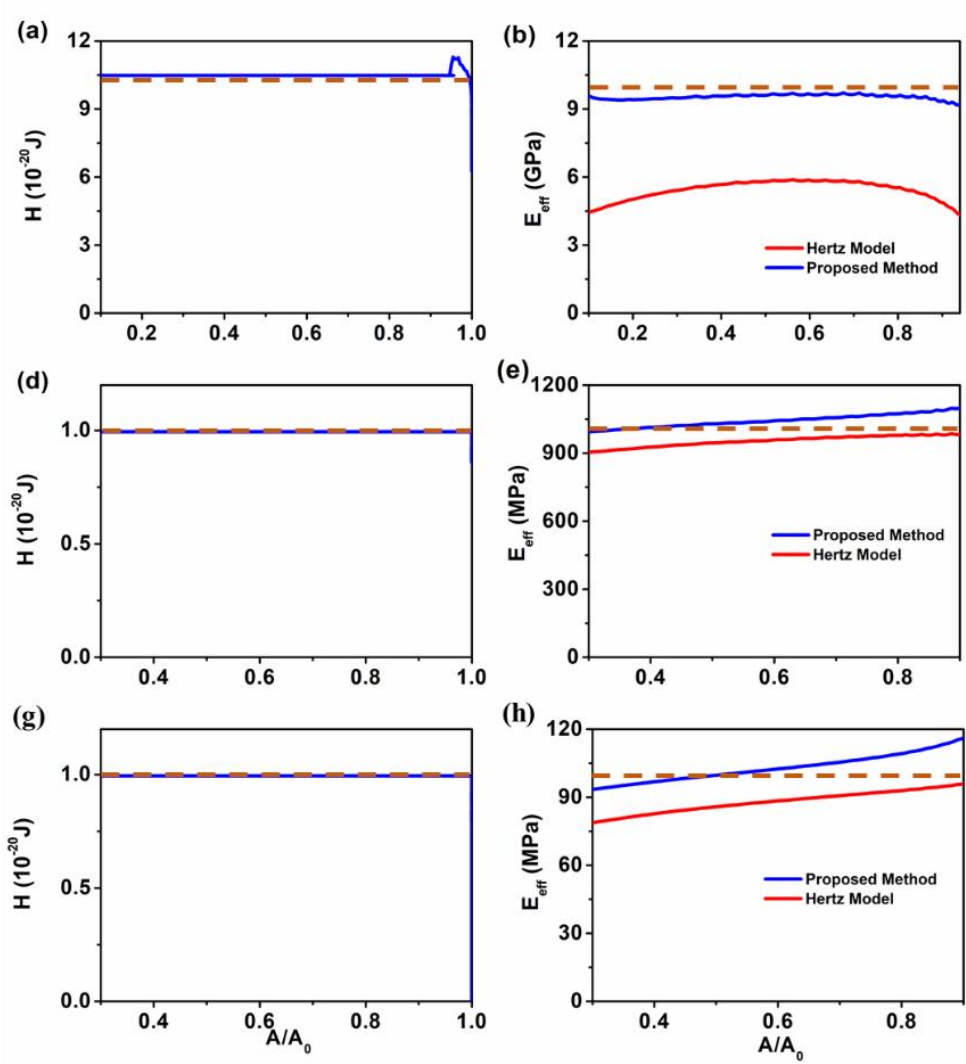
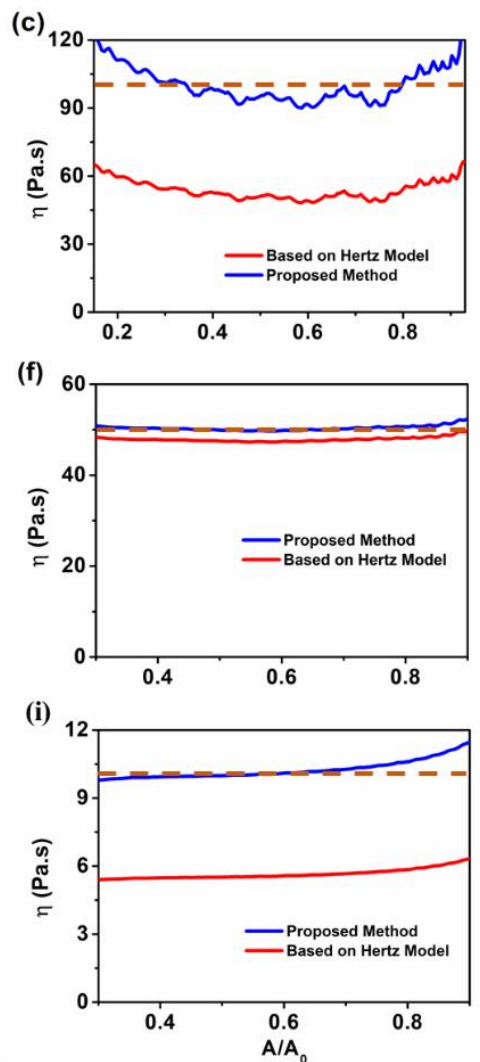

Fig.2. Simulation results and calculations for Cases 1-3 (rigid to compliant materials). The dashed lines are simulated material's set values for Hamaker constant, Young modulus and coefficient of viscosity. Case 1: a) calculated Hamaker constant, b) Young modulus calculated by present method and by derivation from Hertz model, c) calculated viscosity coefficient by present method and by using the Young modulus calculated by Hertz model - named in the graph as 'Based on Hertz model'. Case 2: d) calculated Hamaker constant, e) Young modulus calculated by present method and by derivation from Hertz model, f) calculated viscosity coefficient by present method and by derivation Young modulus from Hertz model. Case 3: g) calculated Hamaker constant, h) Young modulus calculated by present method and by derivation from Hertz model, i) calculated viscosity coefficient by present method and by derivation Young modulus from Hertz model. The parameters of simulations for case 1 are $E_{\text {eff }}=10 \mathrm{GPa}, \mathrm{H}=10^{-19} \mathrm{~J}, \eta=100 \mathrm{~Pa}$. $\mathrm{s}$. For case 2 are $E_{\text {eff }}=1 \mathrm{GPa}, \mathrm{H}=10^{-20} \mathrm{~J}, \eta=$ 50 Pa.s. For case 3 are $E_{\text {eff }}=100 \mathrm{MPa}, \mathrm{H}=10^{-20} \mathrm{~J}, \eta=10 \mathrm{~Pa}$.

For rigid material (case 1), Hamaker constant, Young modulus and viscosity coefficient are calculated using equations 8 and 13 (figure 2 (a), (b) and (c) respectively). The results show very high accuracy for Hamaker constant with less than $3 \%$ error. To calculate the Young modulus, based on the assumption that $(A \gg \delta)$, the most appropriate region of amplitude lies in the range of $\mathrm{A} / \mathrm{A}_{0}$ between 0.3 and 0.9 . Also, it is worthy to mention that, being Young modulus and viscosity calculated over the repulsive regime only, the range of $\mathrm{A} / \mathrm{A}_{0}$ in figures 2 (b) and (c) is limited to the value corresponding to the transition from attractive to repulsive regime and the values in repulsive regime. The very fact that Hertz model derivation (e.g. from equation 31 of ${ }^{31}$ ) yields an expression with only first term of equation 7 , reveals the superiority of the proposed equation. In fact, Hertz model neglects the effect of adhesion (which on the contrary is considered in the proposed method by including the Hamaker constant), which affects the accuracy of the resulting Young modulus values and subsequently - due to the dependence of viscosity coefficient on the value of Young modulus (equation 13) - affects the precision of the calculated values of the viscoelastic parameters of materials. 
The simulation results for semi-rigid material (case 2) are given in figure 2 (d)-(f). To calculate the effective Young modulus and viscosity the most accurate region of $\mathrm{A} / \mathrm{A}_{0}$ lies between 0.4 and 0.9 . Also, the accuracy of the proposed analytical method to calculate the Hamaker constant is depicted in figure 2 (d). A comparison between Young modulus calculated from the presented method and Hertz model (figure 2 (e)) evidences the higher accuracy of the analytical equation 7 , which consequently yields higher accuracy in the calculation of viscosity from equation 13 .

Simulation on compliant material (Case 3) have been performed with Hamaker constant value as for Case 2, and with Young modulus of $100 \mathrm{MPa}$. Again, as for the previous cases, the calculated Hamaker constant, viscosity and Young modulus have high accuracy, witnessing the superiority of the proposed method with respect to the methods based on Hertz model.

Generally, comparison of the calculated Young modulus of figures 2 (b), (e) and (h), shows that by decreasing Young modulus and increasing the indentation length, there is a small dependence between amplitude ratio and calculated Young modulus. For a small amplitude ratio, the calculated Young modulus decreases, which is related to the increasing indentation at small amplitude values. Therefore, based on the assumption used to derive equation 7, when the value of indentation length is comparable to the amplitude, the accuracy of calculated Young modulus is reduced, which directly affects the accuracy of calculated viscosity (equation 13). However, as it can be appreciated by figures 2 (b), (e) and (h), calculation of Young modulus as by present method yields values more reliable than the ones obtained by Hertz model. This fact shows the importance of including Hamaker constant value in the calculation of Young modulus (second term of equation 7), especially in cases where the sample is highly adhesive. Consequently, an increase in value of the Hamaker constant (Case 2 and 3 with respect to Case 1), leads to a larger difference between results from Hertz model and presented method.

The accuracy of the derived expressions to calculate Young modulus, Hamaker constant and viscosity can be directly evaluated by comparing the calculated values with those set in the simulation for model force, resulting in a remarkable agreement between them (figure (S4) and table S1). As well, we have evaluated the error arising from the calculation of different values of one parameter while keeping the other two fixed (figure (S4) and table S1). Finally, we evaluate the accuracy of the proposed method for different free amplitude and resonance frequency values (table S2). It resulted that the maximum errors in the calculations occur with compliant material for Young modulus, with less viscous sample for viscosity and with highest van der Waals force for Hamaker constant. As well, it is noteworthy that the error in Young modulus decreases with increasing material's stiffness, which is explained by the fact that the stiffer the material, the smaller the indentation length with respect to the amplitude. From the results of figure (S4) and Table S1, it can be summarized that calculations for rigid material with low adhesion yield less error in comparison with compliant materials with high adhesion. More details of error analysis are given in Supplementary Information.

To further validate the accuracy of the proposed method, amplitude and phase curves have been experimentally acquired on LDPE and PS as test samples, from which the values Young modulus, Hamaker constant, viscosity and loss tangent of the materials at the nanoscale were extracted. We have performed spectroscopy measurements using AM-AFM on different points of a Polystyrene sample. The amplitude and phase versus 
minimum distance of three sets of measurements are shown in figure 3(a) and (b). The values of Hamaker constant, Young modulus and viscosity coefficient extracted from one of the measurements are given in figure 3(e), (f) and (g). To check the accuracy of extracted parameters of interaction force from our analytical equations, we have used the parameters to reconstruct the force according to the DMT force model and compare the recovered force with the measured one from numerical integral method ${ }^{44}$ (figure $3(\mathrm{c})$ ). As it can be seen there is a good agreement between both analytical and numerical methods in the reconstruction the force. Since the developed equations to extract the parameters is based on the DMT model, the reconstructed force from analytical method only consider vdW force in the attractive regime. It means the effect of capillary force is neglected in our analytical force reconstruction approach which makes a slightly difference with the measured force from numerical method at the initial steps of attractive regime. As it is obvious, the calculated values of Young modulus, Hamaker constant and coefficient of viscosity, shown for one measurement in figure 3(d) to (f) and in their totality in figure 5, supports the validity of our equations, as will be discussed in the following.
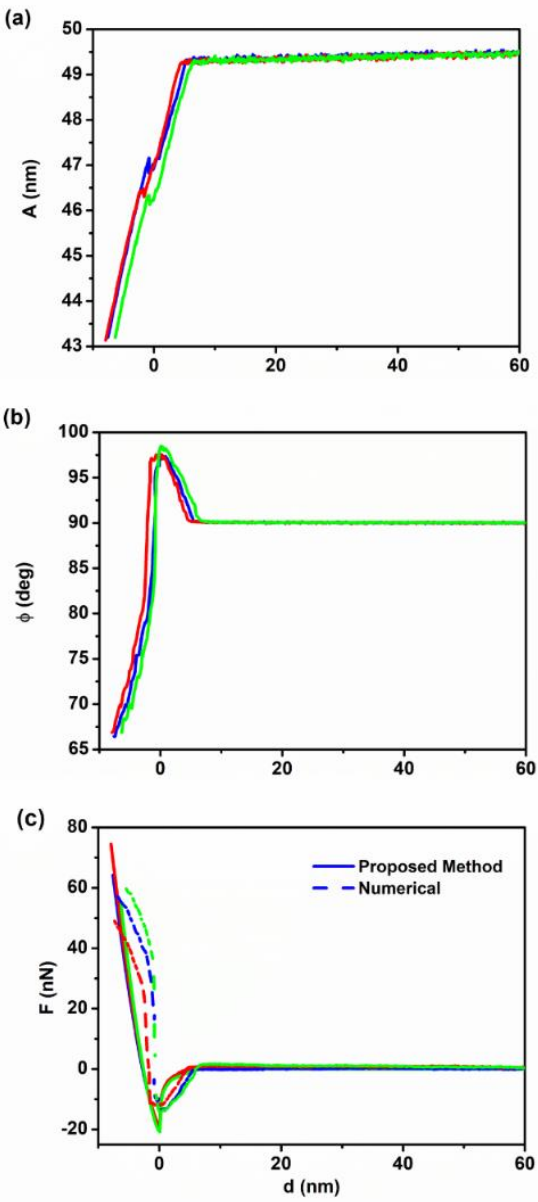
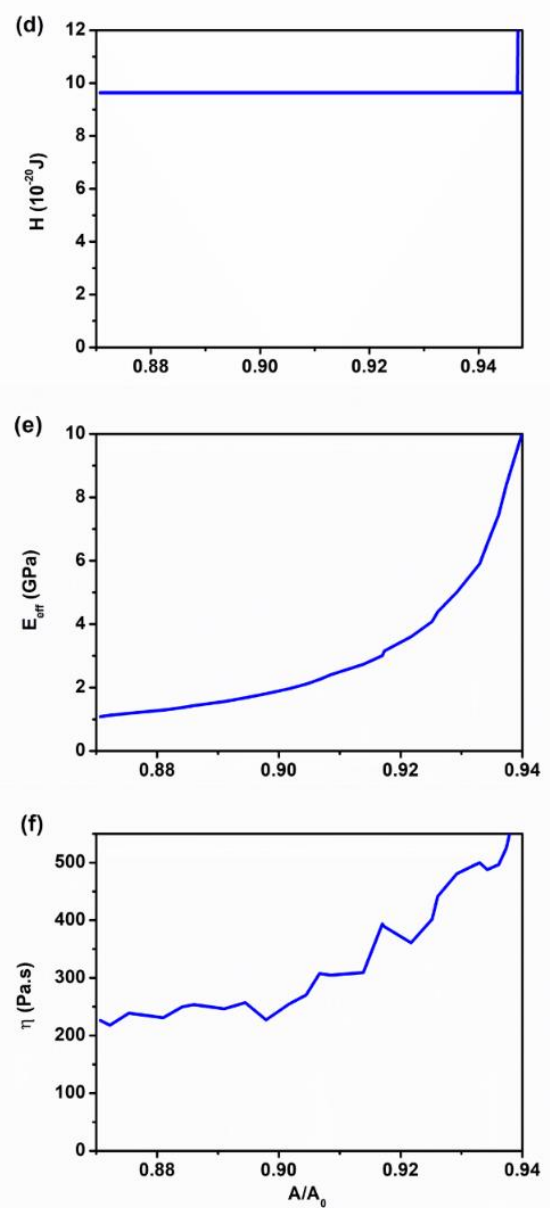

Figure 3. Extrapolation of Polystyrene properties by use of the analytical method. a) amplitude versus minimum distance for three sets of measurements, b) phase versus minimum distance, c) force curves reconstructed from numerical and analytical methods. Resulting properties versus amplitude ratio: d) Young modulus, e) Hamaker constant, f) coefficient of viscosity.

The calculated values of Hamaker constant, Young modulus, viscosity and tangent loss from measurements on PS are presented as histograms in figure 4. The Young modulus ranges between $1 \mathrm{GPa}$ to $4.5 \mathrm{GPa}$, with mean value of $2.8 \mathrm{GPa}$. Nanoindentation measurements gave a Young modulus value of $4.6 \pm 0.2 \mathrm{GPa}$. The difference 
in $E_{\text {eff }}$ value could be because of surface forces, significant for the shallow depth AFM measurements, as opposed to the nanoindentation measurements carried out at $2000 \mathrm{~nm}$ depth. The mean value obtained for Hamaker constant is $13 \times 10^{-20} \mathrm{~J}$ ), while values reported in literature ${ }^{10}$ for effective Hamaker constant between Silicon and PS lie in the range $13-16 \times 10^{-20}(\mathrm{~J})$ which validate the high accuracy of proposed equation. We should note that in AFM measurement the effective Hamaker constant should be considered, which is calculated by the combination of interactions between silicon (the cantilever material), air (the medium) and PS. The variation in the measured values is not surprising due to the broad data range of the experimental data as can be seen in figures 3(a) and (b) and different features and topography changes of the sample surface as seen in figure 4(a). The mean value for loss tangent of PS is 0.1 (figure 4(d)), which is in remarkable agreement with the measured values from ${ }^{16,17}$. The viscosity coefficient (figure $4(\mathrm{e})$ ) is obtained as 300 Pa.s, and the only reference calculating the viscosity coefficient for PS using Kelvin-Voigt model for AFM measurements ${ }^{20}$ considers compressive viscosity coefficient in the modeling of non-conservative force, while the one used for the proposed model is twice the value. Nonetheless, taking in account the differences, the values show good agreement (the details of comparison are given in Supplementary Information).

(a)
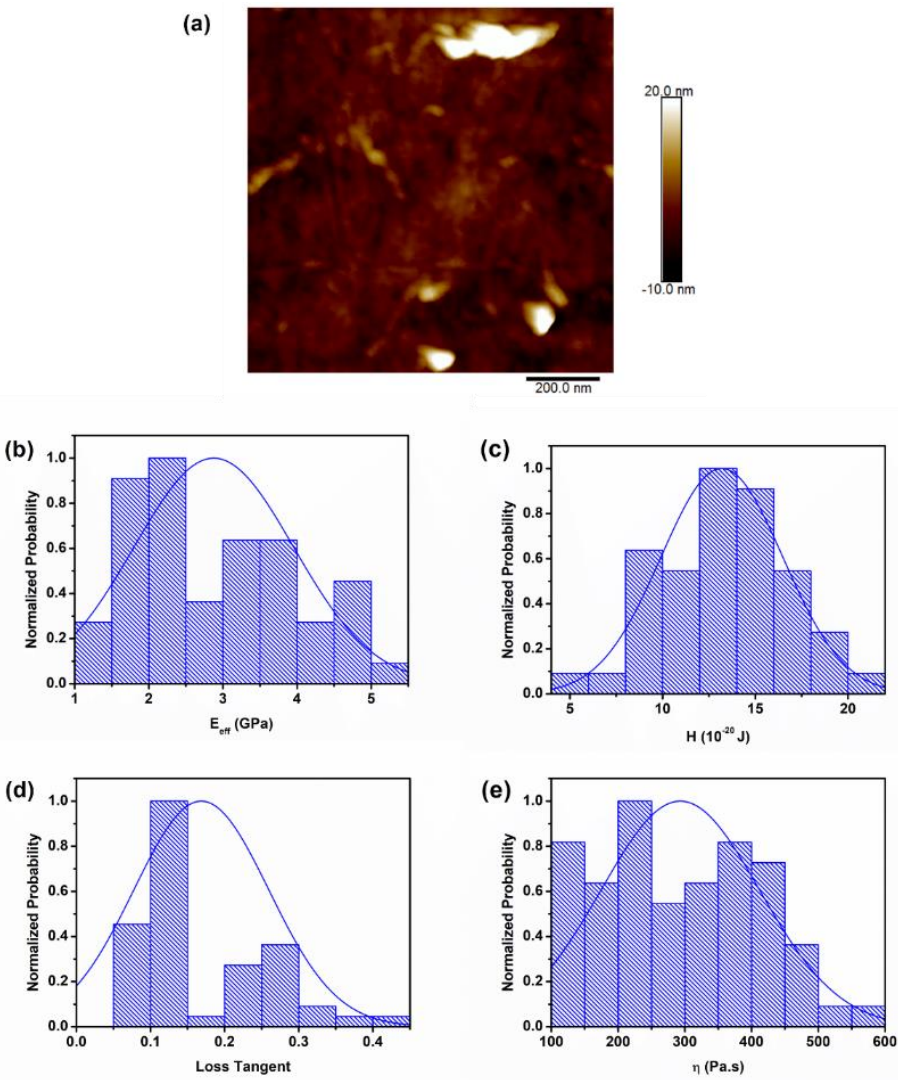

Fig. 4. Experimental results of AM-AFM for PS. a) topography of the area on which the spectroscopy measurements have been performed. Histogram of calculated b) Young modulus, c) Hamaker constant, d) loss tangent and e) coefficient of viscosity.

Figure 5(a) shows the image of the topography of LDPE obtained by tapping mode AFM. The results of quantitative analysis of measured values of Hamaker constant and viscoelastic properties of LDPE are given in figure 5(b)-(e). The measured values for Young modulus lie between $100 \mathrm{MPa}$ and $500 \mathrm{MPa}$, with mean value of $220 \mathrm{MPa}$. Nanoindentation measurements on the same sample gave a Young modulus value of $0.3 \pm 0.1 \mathrm{GPa}$, broadly in agreement with the AFM measurement. The effective Hamaker constant - resulting from the 
integration of silicon (the cantilever material), air (the medium) and LDPE - has a mean value of $11.5 \times 10^{-20}(J)$. Reported values of effective Hamaker constant between Silicon and LDPE ${ }^{41,49,50},{ }^{10}$ are around $16 \times 10^{-20}(\mathrm{~J})$. As it is clear, there is a good agreement between the measured and reported values considering the fact that the smaller value of measurement can be attributed to the effect of oxidization of Silicon tip which slightly reduces its effective Hamaker constant. Therefore, by considering the mentioned factors and the spread of the obtained values, the differences with literature are acceptable and using the proposed equations the Hamaker constant can be calculated with high accuracy. The mean value for loss tangent of LDPE is 0.6 , in agreement with the ones from ${ }^{20}$. The measured viscosity coefficient for LDPE is obtained as 150 Pa.s. comparable to reported values obtained by AFM ${ }^{20}$. As for PS, considering the fact that in our model the coefficient of viscosity is twice the value of ${ }^{20}$, the value measured in ${ }^{20}$ should be compared using the same conditions used for the present work as explain with details in Supplementary Information. The fact that the obtained value for Young modulus and coefficient of viscosity based on our method are higher than the reported values in ${ }^{20}$ can be explained by the mechanism of calculation of Young modulus. The measured Young modulus in our method is $220 \mathrm{MPa}$ while in ${ }^{20}$ is reported around $110 \mathrm{MPa}$, since in ${ }^{20} \mathrm{Hertz}$ model is used, which neglect the effect of adhesion. As already shown, this assumption leads to underestimate the Young modulus, especially in the case that the Hamaker constant is significant. Hence it can be concluded that the value for viscosity and Young modulus calculated by the proposed method have high accuracy, which is supported by the agreement between the Young modulus given by the analytical method and by nanoindentation measurements.

(a)
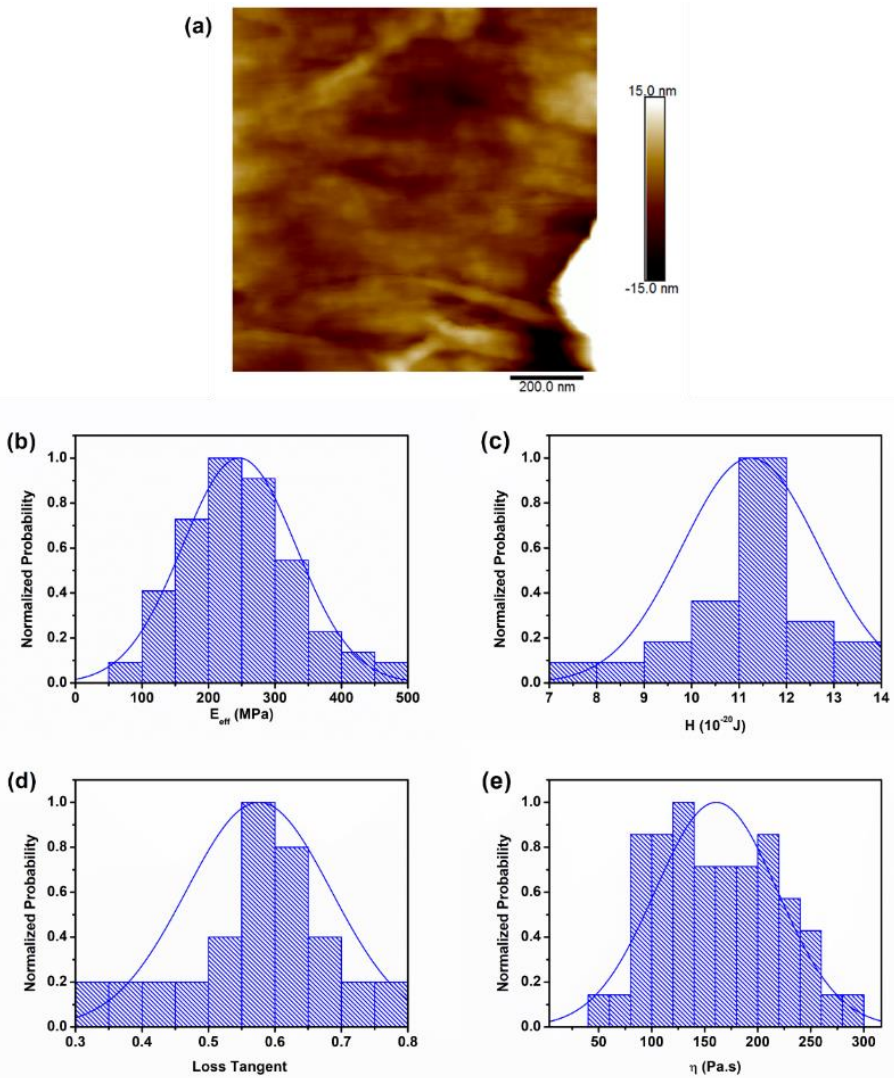

Fig. 5. Experimental results of AM-AFM for LDPE. a) topography of the area on which the spectroscopy measurements have been performed. Histogram of calculated b) Young modulus, c) Hamaker constant, d) loss tangent and e) coefficient of viscosity. 
Finally, to verify the accuracy of our method to determine viscoelastic properties of materials in relation to tip radius of curvature uncertainty, in figure 6 we compare dissipation and virial obtained from the numerical simulation based on the parameters of experiments with the ones directly obtained by the experimental data of LDPE. For the LDPE, we choose the range measured values from our proposed method $\left(\mathrm{E}_{\mathrm{LDPE}}=220 \mathrm{MPa}\right.$, $\mathrm{H}_{\mathrm{LDPE}}=6 \times 10^{-20} \mathrm{~J}$ and viscosity=100 Pa.s). To evaluate the effect of tip radius on the results, we consider different uncertainty values in the tip radius and simulation. As it is depicted, for the energy dissipation, the effect of higher tip radius is more significant than the lower tip radius especially for the amplitude ratio between 1 to 0.6. After $\mathrm{A} / \mathrm{A}_{0}=0.6$, the tip radius does not affect the simulation and the simulated results are fitted very well with experiments. For the case of virial, both decrease and increase of tip radius affect the accuracy of simulation. However, especially for nominal tip radius, the trend of viral for both simulation and experiments fitted very well.

To consider the uncertainty in the values of some parameters of our measurement such as free amplitude, spring constant and quality factor of cantilevers, results from several experiments are displayed. Looking at the dissipated energy, which is really a signature of the repulsive region were contact does occur, the agreement between the model and the data is for low $\mathrm{A} / \mathrm{A}_{0}$ ratio, where indeed the predominance of repulsive interactions is significant. At high $\mathrm{A} / \mathrm{A}_{0}$ ratio, the tip is increasingly in the attractive regime where other interactions, such as capillary and electrostatic interactions may play a role, not taken into account by the model. The same comment can be made about the virial energy which incorporate all interactions.

(a)

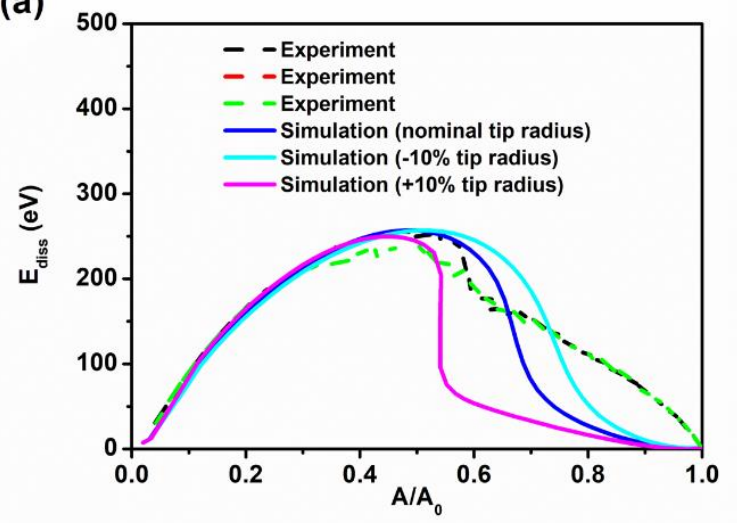

(b)



Figure 6. Comparison between numerical simulation and experimental extrapolation from data on LDPE. a) energy dissipation and b) the virial calculated for three different values of tip radius.

In order to properly evaluate the accuracy and ability of the proposed analytical equations to extract materials properties, two main issues should be addressed.

The first issue is related to the calculation of loss tangent and coefficient of viscosity. As we mentioned in the equations 13 and 14 the viscoelastic parameters that characterize the response of a material might be frequency dependent. Therefore, in an AFM measurement, the coefficient viscosity might depend on the frequency of the oscillating tip and by changing the frequency of cantilever this value can be changed. Also, it should be noted, 
our non-conservative force model based on Kelvin-Voigt model is an approximate model considering fixed strain, hence it fails to describe stress relaxation. However, this fact does not affect the accuracy of our model and extrapolation method. Given the dynamic origin of the AFM measurements, which apply a time-dependent force on the sample, the indentation length is a function of cantilever frequency and, due to the sinusoidal motion of cantilever it has no fixed value. Therefore, as mentioned in ${ }^{20}$, for dynamic AFM experiments the assumption of linear viscoelastic regime is valid. As well, considering the proposed method the effect of adhesion in calculating the Young modulus - in contrast with the Hertz model, for which increasing indentation leads to larger error - this source of error is reduced.

The second issue is related to the importance of the tip radius in the parameters' quantification. To quantify the nanomechanical and chemical properties of samples, the value of tip radius must be known. As discussed above, a change of tip radius could affect the accuracy of quantification especially for the calculation of Hamaker constant. The procedure used to calculate the tip radius is based on tip shape deconvolution by blind reconstruction method ${ }^{51}$ which lacks a rigorous uncertainty estimation for the obtained value of radius of curvature. However, in first place the radius of curvature (RoC) value obtained by this procedure represents an upper boundary of the actual physical radius. Secondly, by using a range of results depending on expected size and sampled area parameters to obtain an averaged tip radius, the resulting standard deviation lies well below $10 \%$, which is in line with the uncertainty used for the calculations from experimental data.

\section{Conclusion}

In the present work we introduce a fully analytical method able to extrapolate Hamaker constant and viscoelastic properties of materials at the nanoscale using tapping mode AFM. The only measured data are amplitude and phase versus average distance between probe and surface which are easily accessible by using any standard commercial AFM systems in AM-AFM mode. The accuracy of the derived expressions to calculate Young modulus, Hamaker constant and viscosity has been evaluated against the values set in the simulation for model force, resulting in a remarkable agreement, and performing better than Hertz model. Validation on AM-AFM experimental data from LDPE and PS test samples confirmed the accuracy of the proposed method, with obtained values matching the reported ones and the ones obtained by nanoindentation experiments. Furthermore, data from simulation and experiments proved the robustness of the method towards variation in cantilever's amplitude and frequency and uncertainty in tip radius of curvature. The superiority of the proposed method to the ones currently used in the AFM community lies in the inclusion of adhesion to calculate Young modulus and viscoelasticity, and it is applicable for materials with properties ranging from rigid to soft, adhesive to non-adhesive and viscous to non-viscous. The proposed equation to calculate Young modulus can be used for determination of stiffness of any material and can be extended for other advanced AFM methods.

\section{Acknowledgements}

We would like to acknowledge the funding support from Invest N. Ireland (RD0714186) for AM.

\section{References}


1. Block, J. et al. Viscoelastic properties of vimentin originate from nonequilibrium conformational changes. Sci. Adv. 4, (2018).

2. Collins, L., Liu, Y., Ovchinnikova, O. S. \& Proksch, R. Quantitative Electromechanical Atomic Force Microscopy. ACS Nano 13, 8055-8066 (2019).

3. Essmann, C. L. et al. Mechanical properties measured by atomic force microscopy define health biomarkers in ageing C. elegans. Nat. Commun. 11, (2020).

4. Garcia, R. Intracellular forces from stiffness. Nat. Mater. 18, 1037-1038 (2019).

5. Nishizawa, K. et al. Feedback-tracking microrheology in living cells. Sci. Adv. 3, 1-13 (2017).

6. $\quad$ Piantanida, L., Payam, A. F., Zhong, J. \& Voïtchovsky, K. Nanoscale mapping of the directional flow patterns at liquid-solid interfaces. Phys. Rev. Appl. (2020).

7. Uhlig, M. R. \& Magerle, R. Unraveling capillary interaction and viscoelastic response in atomic force microscopy of hydrated collagen fibrils. Nanoscale $9,1244-1256$ (2017).

8. Das, S., Sreeram, P. A. \& Raychaudhuri, A. K. A method to quantitatively evaluate the Hamaker constant using the jump-into-contact effect in atomic force microscopy. Nanotechnology 18, (2007).

9. Fronczak, S. G., Browne, C. A., Krenek, E. C., Beaudoin, S. P. \& Corti, D. S. Non-contact AFM measurement of the Hamaker constants of solids: Calibrating cantilever geometries. J. Colloid Interface Sci. 517, $213-220$ (2018).

10. Leite, F. L., Bueno, C. C., Da Róz, A. L., Ziemath, E. C. \& Oliveira, O. N. Theoretical models for surface forces and adhesion and their measurement using atomic force microscopy. International Journal of Molecular Sciences 13, (2012).

11. Lomboy, G., Sundararajan, S., Wang, K. \& Subramaniam, S. A test method for determining adhesion forces and Hamaker constants of cementitious materials using atomic force microscopy. Cem. Concr. Res. 41, 1157-1166 (2011).

12. Miklavcic, S. J. Reliable and accurate extraction of Hamaker constants from surface force measurements. J. Colloid Interface Sci. 524, 263-266 (2018).

13. Al-Rekabi, Z. \& Contera, S. Multifrequency AFM reveals lipid membrane mechanical properties and the effect of cholesterol in modulating viscoelasticity. Proc. Natl. Acad. Sci. U. S. A. 115, 2658-2663 (2018).

14. Amo, C. A., Perrino, A. P., Payam, A. F. \& Garcia, R. Mapping Elastic Properties of Heterogeneous Materials in Liquid with Angstrom-Scale Resolution. ACS Nano 11, 8650-8659 (2017).

15. Lai, C. Y., Santos, S. \& Chiesa, M. Systematic Multidimensional Quantification of Nanoscale Systems from Bimodal Atomic Force Microscopy Data. ACS Nano 10, 6265-6272 (2016).

16. Proksch, R. et al. Practical loss tangent imaging with amplitude-modulated atomic force microscopy. J. Appl. Phys. 119, (2016).

17. Yablon, D. G., Grabowski, J. \& Chakraborty, I. Measuring the loss tangent of polymer materials with atomic force microscopy based methods. Meas. Sci. Technol. 25, (2014).

18. Thorén, P. A. et al. Modeling and Measuring Viscoelasticity with Dynamic Atomic Force Microscopy. Phys. Rev. Appl. 10, 1 (2018).

19. Rajabifar, B., Jadhav, J. M., Kiracofe, D., Meyers, G. F. \& Raman, A. Dynamic AFM on Viscoelastic Polymer Samples with Surface Forces. Macromolecules 51, 9649-9661 (2018).

20. Benaglia, S., Amo, C. A. \& Garcia, R. Fast, quantitative and high resolution mapping of viscoelastic properties with bimodal AFM. Nanoscale 11, 15289-15297 (2019).

21. Benaglia, S., Gisbert, V. G., Perrino, A. P., Amo, C. A. \& Garcia, R. Fast and high resolution mapping of elastic properties of biomolecules and polymers with bimodal AFM Simone. Nat. Protoc. 13, 2890-2907 (2018).

22. Claesson, P. M. et al. From force curves to surface nanomechanical properties. Phys. Chem. Chem. Phys. 19, 23642-23657 (2017).

23. Crippa, F. et al. Probing nano-scale viscoelastic response in air and in liquid with dynamic atomic force microscopy. Soft Matter 14, 3998-4006 (2018).

24. Dietz, C., Schulze, M., Voss, A., Riesch, C. \& Stark, R. W. Bimodal frequency-modulated atomic force microscopy with small cantilevers. Nanoscale 7, 1849-1856 (2015).

25. Efremov, Y. M., Cartagena-Rivera, A. X., Athamneh, A. I. M., Suter, D. M. \& Raman, A. Mapping heterogeneity of cellular mechanics by multi-harmonic atomic force microscopy. Nat. Protoc. 13, 2200-2216 (2018).

26. Efremov, Y. M., Wang, W. H., Hardy, S. D., Geahlen, R. L. \& Raman, A. Measuring nanoscale viscoelastic parameters of cells directly from AFM force-displacement curves. Sci. Rep. 7, 1-14 (2017).

27. Herruzo, E. T., Perrino, A. P. \& Garcia, R. Fast nanomechanical spectroscopy of soft matter. Nat. Commun. 5 , (2014).

28. Lai, C. Y., Perri, S., Santos, S., Garcia, R. \& Chiesa, M. Rapid quantitative chemical mapping of surfaces with sub$2 \mathrm{~nm}$ resolution. Nanoscale 8, 9688-9694 (2016).

29. Garcia, R. \& Proksch, R. Nanomechanical mapping of soft matter by bimodal force microscopy. Eur. Polym. J. 49, 1897-1906 (2013).

30. Bielefeldt, H. \& Giessibl, F. J. A simplified but intuitive analytical model for intermittent-contact-mode force microscopy based on Hertzian mechanics. Surf. Sci. 440, (1999).

31. Hölscher, H. \& Schwarz, U. D. Theory of amplitude modulation atomic force microscopy with and without QControl. Int. J. Non. Linear. Mech. 42, 608-625 (2007).

32. García, R., Magerle, R. \& Perez, R. Nanoscale compositional mapping with gentle forces. Nat. Mater. 6, 405-411 (2007).

33. García, R. \& San Paulo, A. Amplitude curves and operating regimes in dynamic atomic force microscopy. Ultramicroscopy 82, 79-83 (2000).

34. Paulo, Á. S. \& García, R. Tip-surface forces, amplitude, and energy dissipation in amplitude-modulation (tapping 
mode) force microscopy. Phys. Rev. B - Condens. Matter Mater. Phys. 64, 1-4 (2001).

35. San Paulo, A. \& García, R. High-resolution imaging of antibodies by tapping-mode atomic force microscopy: Attractive and repulsive tip-sample interaction regimes. Biophys. J. 78, 1599-1605 (2000).

36. Kim, D. \& Sahin, O. Imaging and three-dimensional reconstruction of chemical groups inside a protein complex using atomic force microscopy. Nat. Nanotechnol. 10, 264-269 (2015).

37. Legleiter, J., Park, M., Cusick, B. \& Kowalewski, T. Scanning probe acceleration microscopy (SPAM) in fluids: Mapping mechanical properties of surfaces at the nanoscale. Proc. Natl. Acad. Sci. U. S. A. 103, 4813-4818 (2006).

38. Santos, S., Gadelrab, K., Font, J. \& Chiesa, M. Single-cycle atomic force microscope force reconstruction: Resolving time-dependent interactions. New J. Phys. 15, (2013).

39. Stark, M., Stark, R. W., Heckl, W. M. \& Guckenberger, R. Inverting dynamic force microscopy: From signals to time-resolved interaction forces. Proc. Natl. Acad. Sci. U. S. A. 99, 8473-8478 (2002).

40. $\mathrm{Hu}, \mathrm{S}$. \& Raman, A. Inverting amplitude and phase to reconstruct tip-sample interaction forces in tapping mode atomic force microscopy. Nanotechnology 19, (2008).

41. Katan, A. J., Van Es, M. H. \& Oosterkamp, T. H. Quantitative force versus distance measurements in amplitude modulation AFM: A novel force inversion technique. Nanotechnology 20, (2009).

42. Platz, D., Forchheimer, D., Tholén, E. A. \& Haviland, D. B. The role of nonlinear dynamics in quantitative atomic force microscopy. Nanotechnology 23, (2012).

43. Platz, D., Forchheimer, D., Tholén, E. A. \& Haviland, D. B. Interaction imaging with amplitude-dependence force spectroscopy. Nat. Commun. 4, (2013).

44. Payam, A. F., Martin-Jimenez, D. \& Garcia, R. Force reconstruction from tapping mode force microscopy experiments. Nanotechnology 26, 1-12 (2015).

45. Lee, M. \& Jhe, W. General theory of amplitude-modulation atomic force microscopy. Phys. Rev. Lett. 97, 1-4 (2006).

46. Lozano, J. R. \& Garcia, R. Theory of phase spectroscopy in bimodal atomic force microscopy. Phys. Rev. B Condens. Matter Mater. Phys. 79, 014110 (2009).

47. Payam, A. F. Dynamic modeling and sensitivity analysis of dAFM in the transient and steady state motions. Ultramicroscopy 169, 55-61 (2016).

48. Sader, J. E. et al. A virtual instrument to standardise the calibration of atomic force microscope cantilevers. Rev. Sci. Instrum. 87, (2016).

49. Hubbard, A. T. Encyclopedia of Surface and Colloid Science -, Volume 1. (CRC Press, 2002).

50. Dimitriadis, E. K., Horkay, F., Maresca, J., Kachar, B. \& Chadwick, R. S. Determination of elastic moduli of thin layers of soft material using the atomic force microscope. Biophys. J. 82, 2798-2810 (2002).

51. Villarrubia, J. S. Algorithms for scanned probe microscope image simulation, surface reconstruction, and tip estimation. J. Res. Natl. Inst. Stand. Technol. 102, 425-454 (1997). 